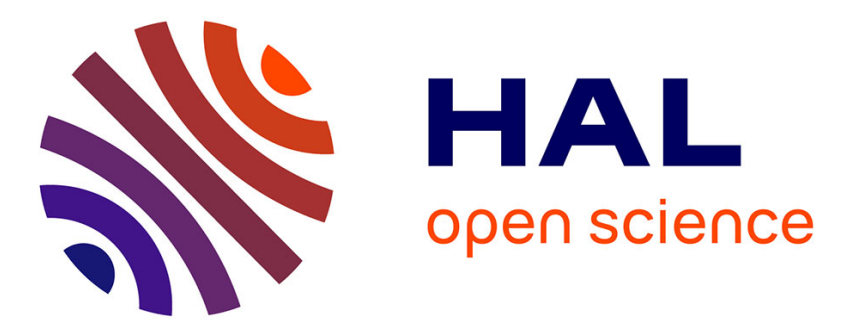

\title{
Registration of Arbitrary Multi-view 3D Acquisitions
}

Camille Chane Simon, Rainer Schutze, Frank Boochs, Franck Marzani

\section{To cite this version:}

Camille Chane Simon, Rainer Schutze, Frank Boochs, Franck Marzani. Registration of Arbitrary Multi-view 3D Acquisitions. Computers in Industry, 2013, 64 (9), pp.1082-1089. hal-00931793

\section{HAL Id: hal-00931793 \\ https://hal.science/hal-00931793}

Submitted on 15 Jan 2014

HAL is a multi-disciplinary open access archive for the deposit and dissemination of scientific research documents, whether they are published or not. The documents may come from teaching and research institutions in France or abroad, or from public or private research centers.
L'archive ouverte pluridisciplinaire HAL, est destinée au dépôt et à la diffusion de documents scientifiques de niveau recherche, publiés ou non, émanant des établissements d'enseignement et de recherche français ou étrangers, des laboratoires publics ou privés. 


\title{
Fusion of Arbitrary Multi-view 3D Acquisitions
}

\author{
Camille Simon Chane ${ }^{\mathrm{a}, \mathrm{b}}$, Rainer Schütze ${ }^{\mathrm{b}}$, Frank Boochs ${ }^{\mathrm{b}}$, Franck S. Marzani ${ }^{\mathrm{a}}$ \\ ${ }^{a}$ le2i, Université de Bourgogne, B.P. 47870, 21078 Dijon, France \\ ${ }^{b}$ i3mainz, Fachhochschule Mainz, Lucy Hillebrand Straße 2, 55128 Mainz, Germany
}

\begin{abstract}
Registration of 3D meshes of smooth surfaces is performed by tracking the acquisition system. The tracking is performed using photogrammetric techniques. Careful calibration of all objects in play enable a registration accuracy of $* * *$. Targets are used to asses the precision of the registration, but the method does not rely on the use of targets and can be used for the registration of featureless surfaces.
\end{abstract}

Keywords: 3D imaging, close-range photogrammetry, 3D registration, multi-view registration

\section{Contents}

1 Introduction 1

2 Materials and Methods 2

2.1 Principle. . . . . . . . . . . . . . . . 2

2.2 Materials . . . . . . . . . . . . . . . . 3

2.3 Calibrations . . . . . . . . . . . . . . . 3

2.4 Data processing . . . . . . . . . . . . . . . 6

3 Results and Discussion $\quad 7$

3.1 Individual calibrations . . . . . . . . . . . . . . . 7

3.2 Tracking the frame . . . . . . . . . . . . 7

3.3 Registration . . . . . . . . . . . . . . . . . . . 9

4 Conclusion and Perspectives $\quad 9$

Email addresses: camille.simon@u-bourgogne.fr (Camille Simon Chane), rainer.schuetze@geoinform.fh-mainz.de (Rainer Schütze),

frank.boochs@geoinform.fh-mainz.de (Frank Boochs), franck.marzani@u-bourgogne.fr (Franck S. Marzani) 


\section{Introduction}

3D imaging is increasingly used for surface inspection. Creating 3D models with contact-less imaging sensors is a greatly useful. However, there are natural trade-offs between the field of view of these devices and their resolution. To scan large objects with a high resolution, multiple acquisitions with complementary fields of view are necessary. These views must then be stitched together to create a complete 3D model. This is the problem of high-accuracy multi-view registration.

In the case of spatially-structured objects, multi-view registration is most often based on the ICP algorithm [1] or one of its variations [2]. This method does not perform well for smooth surfaces and requires a 30\% to $40 \%$ overlap between successive views. On the other hand, basing the registration on feature points requires adequate salient points for each pair of datasets. Targets can be added to the surface if there are no natural salient features, if these features are not well-resolved or if they are insufficient in number. However, it can be tedious and time-consuming to place and remove targets from the multiple objects that we want to digitize. Furthermore, there is always the possibility that a target covers a defect that we want to detect. Also, there may be cases in which we fear damaging the surface with the targets.

Instead of relying on the object data, 3D registration can be based on the known position and orientation of the acquisition system. If a robot is used to control the acquisition system, its' position and orientation is readily available. But the attained accuracy of $1 \mathrm{~mm}$ in untaught mode is not sufficient to base the registration solely on this data. Optical techniques such as photogrammetry can be used to increase robot positioning accuracy via on-line calibration. Two setups are possible: either a camera is fixed to the robot and observes the scene which has been covered with targets $[3,4]$ or the robot is itself covered with targets and observed by a number of fixed cameras $[5,6]$. This second setup is the best suited to be adapted to our task. We can measure the precise position and orientation of the acquisition system in a coordinate system defined by set of fixed cameras which observe the acquisition system the camera by fixing targets to it, instead of on the object under study. Our goal is to register 3D datasets with a precision better than half the acquisition system resolution.

\section{Materials and Methods}

\subsection{Principle}

Figure 1 illustrates the principle of our technique: a set of photogrammetric cameras observe an acquisition system while it digitizes a surface. A targetframe is fixed to the acquisition system to enable the precise tracking of its' position and orientation during the measurements.

The scope of this experiment is to show that this setup can be used to track a $3 \mathrm{D}$ acquisition system and to register the individual 3D acquisitions. The object 


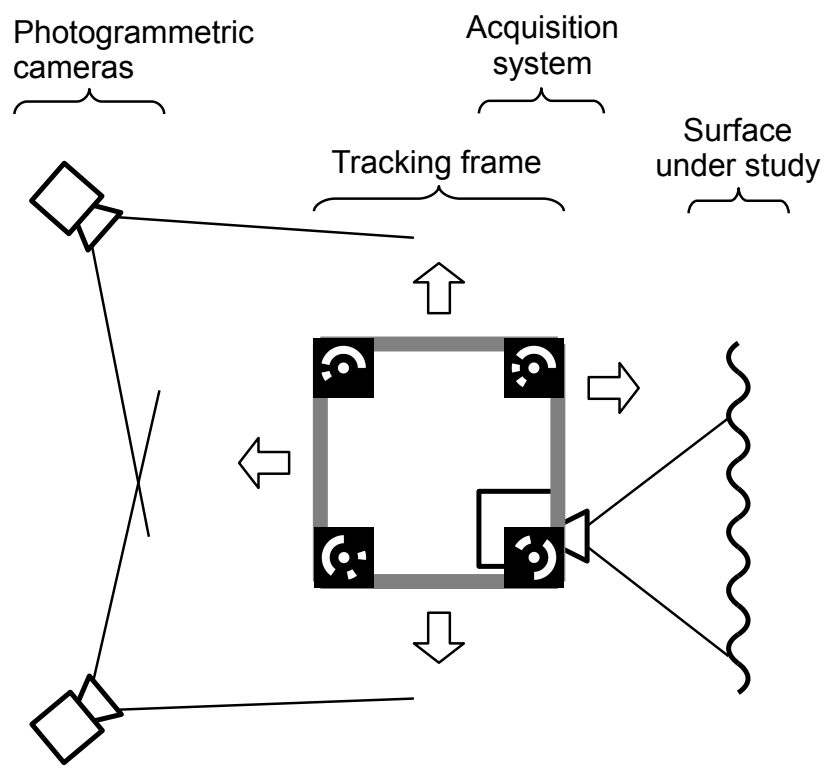

Figure 1: Overview of tracking configuration

under study is a car door which has been covered with un-coded photogrammetric targets. These targets are not used for the tracking and registration but they enable us to measure the accuracy of our registration procedure.

\subsection{Materials}

All 3D digitizations were performed using a commercial fringe projection digitization system by Gom, the Atos III. The system can be built in different field-of-view/ accuracy setups. For this study we use a $500 \mathrm{~mm} \times 500 \mathrm{~mm}$ field of view, yielding an accuracy of $0.24 \mathrm{~mm}$. In this configuration the measuring distance between the Gom Atos III and the surface under study must be $760 \mathrm{~mm}$. This entails that our registration accuracy goal of half the acquisition resolution is $0.12 \mathrm{~mm}$ spatially and $0.158 \mathrm{mrad}$.

The characteristics of the materials used for the tracking procedure were optimized through several simulations, some of which are described in [7]. The four tracking cameras are 5 Mpx AVT Stingray and are used in conjunction with $8 \mathrm{~mm}$ Pentax lens. The tracking frame is an aluminum cube of edge length $500 \mathrm{~mm}$ and covered with 78 targets (see figure 2). A hexagonal headplate is attached to the bottom of the frame so that it can be fixed to a tripod. A hexagonal plate holder inside the cube on the bottom face is used to fix the acquisition system to the frame. An additional camera, a Nikon D300, is used for certain calibration procedures. 


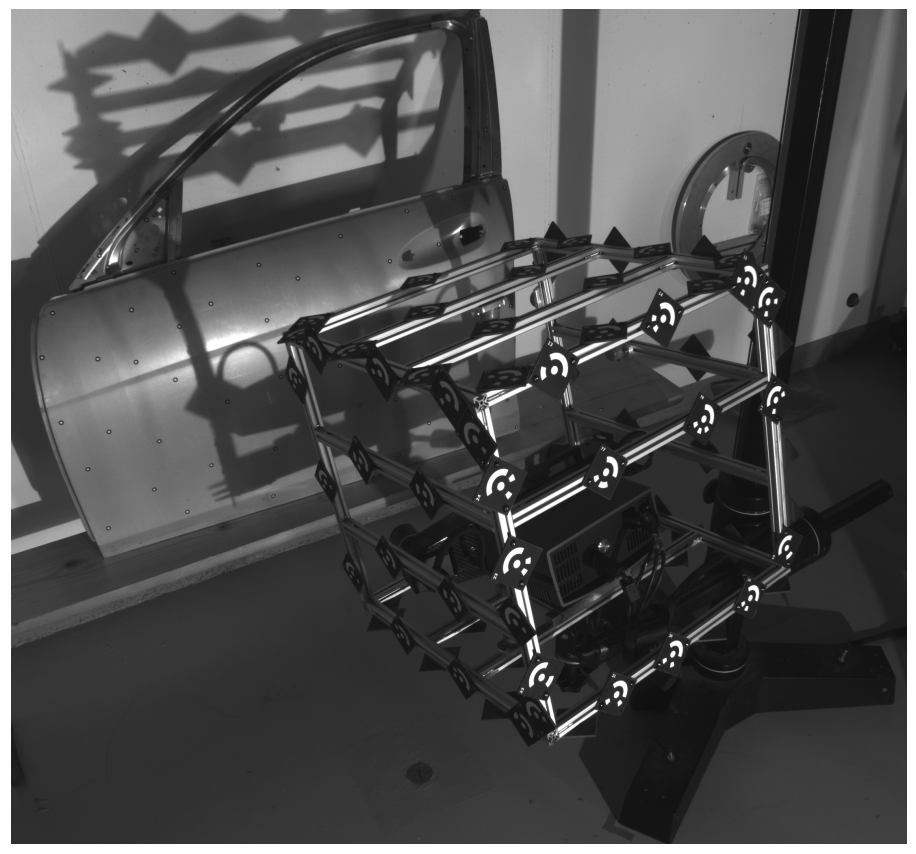

Figure 2: Tracking cube covered with 78 targets with the acquisition system fixed inside the cube. The car door is visible in the background.

\subsection{Calibrations}

To ensure a precise tracking, all optics and objects in play must be carefully calibrated. We thus introduce the following coordinate systems, linked to the materials in use (illustrated figure 3):

- $C_{S},\left(O_{S}, \vec{x}_{S}, \vec{y}_{S}, \vec{z}_{S}\right)$ is the coordinate system linked to the acquisition system.

- $C_{F},\left(O_{F}, \vec{x}_{F}, \vec{y}_{F}, \vec{z}_{F}\right)$ is the coordinate system linked to the tracking frame.

- $C_{C i},\left(O_{C i}, \vec{x}_{C i}, \vec{y}_{C i}, \vec{z}_{C i}\right)$ are the coordinate systems linked to the each tracking camera. $O_{C i}$ is the optical center of the camera, $\left(\vec{x}_{C i}, \vec{y}_{C i}\right)$ define the image plane, $\vec{z}_{C i}$ is collinear to the optical axis.

- $C_{0},\left(O_{0}, \vec{x}_{0}, \vec{y}_{0}, \vec{z}_{0}\right)$ is the world coordinate system.

We now describe the necessary calibrations and how they are performed. The following notations will be used throughout this section: $\left.A\right|_{C_{U}}$ are the homogeneous coordinates $\left(x_{A}, y_{A}, z_{A}, 1\right)$ of point $A$ in coordinate system $C_{U}$. We define $T_{C_{U}, C_{V}}$ the transformation matrix between two coordinate system $C_{U}$ and $C_{V}$ such that $\left.T_{C_{U}, C_{V}} \cdot A\right|_{C_{V}}=\left.A\right|_{C_{U}}$. 


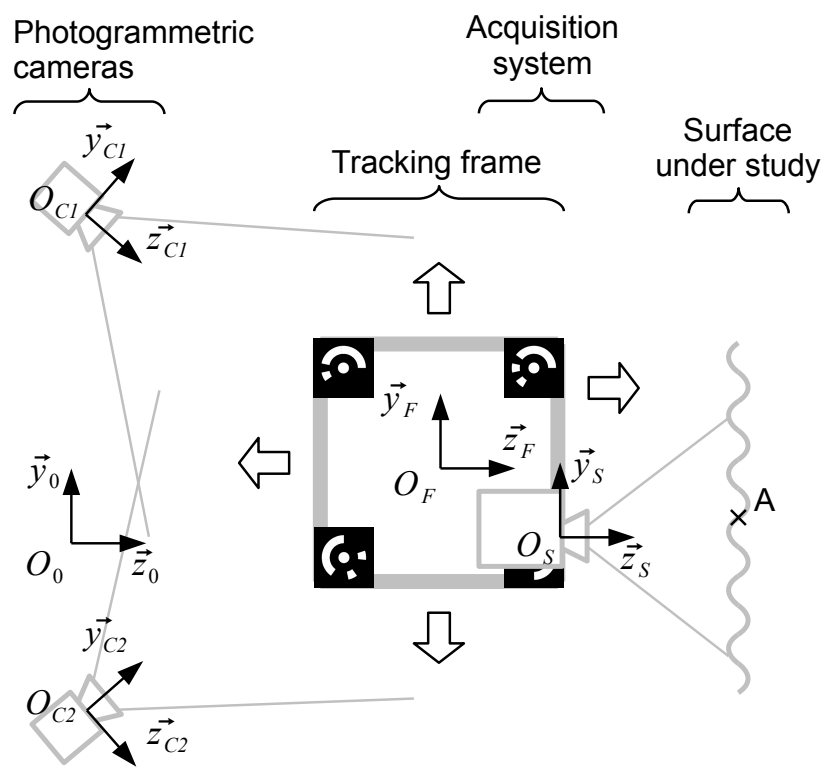

Figure 3: Coordinate systems defined for the tracking procedure.

Internal orientation (I.O.) of the photogrammetric cameras. The calibration of the tracking cameras is performed by taking close to a hundred images of a calibration plate from various points of view. The calibration plate is covered with coded and uncoded targets and two distances are precisely known. From this we can measure the internal camera parameters such as focal length, principle point offset and lens distortion. The four tracking cameras are placed side by side and observe the same area so that they can be calibrated together. Previous experience has taught us that the internal orientation can stay stable for over a week if the cameras are handled with care during this time frame. We thus perform the interior orientation a few days before the acquisitions.

Internal orientation of the acquisition system. It is also necessary to know the distortions introduced by the acquisition system. In the case of the Atos III there is a specific calibration procedure to perform and the output data is corrected by the acquisition software.

Calibration of the tracking frame. The cube calibration is performed by taking over one hundred images of the tracking cube with a scale bar and additional targets. We can then define $C_{F}$ and know the position of each coded target in this coordinate system.

Orientation of the acquisition system with respect to the tracking frame. To know the position of the acquisition system in the system defined by the tracking cube, we proceed in three steps: 
1. Fix the acquisition system to the tracking frame.

2. Use the acquisition system to digitize a target-covered 3D object.

3. Take approximately fifty photos of the tracking frame and target $3 \mathrm{D}$ object.

We associate a coordinate system $C_{t} e m p$ to the 3D object. Step 2 provides us with the position and orientation of the $3 \mathrm{D}$ object in $C_{S}$, that is $T_{S, t e m p}$. Similarly, step 3 provides us with the position and orientation of the 3D object in $C_{F}$ previously defined, that is $T_{F, t e m p}$.

We can thus easily calculate the transformation between $C_{S}$ and $C_{F}: T_{F, S}=$ $T_{F, \text { temp }} \cdot\left(T_{S, t e m p}\right)^{-1}$.

Relative orientation of the photogrammetric cameras. Once the tracking cameras have been positioned to observe the area in front of the surface under study, we can measure their relative position and orientation. This is done by acquiring approximately eighty images of a scalebar in various positions and orientations, simultaneously by the four calibrated cameras.

The position and orientation of the other three cameras are measured with respect to the first.

We choose the coordinate system of the first tracking camera as the world system: $C_{0}=C_{C 1}$.

\subsection{Data processing}

We thus have the necessary data to register the data sets. Each acquisition provides us with the coordinates of a group of surface points in the sensor system, $\left.A\right|_{C_{S}}$. The simultaneous tracking provides us with $T_{C_{0}, C_{F}}$. The known interior orientation of the tracking cameras and their relative orientation ensures that $T_{C_{0}, C_{F}}$ is sufficiently precise.

We can thus calculate $\left.A\right|_{C_{0}}$, the coordinates of the surface points in the world system using:

$$
\left.A\right|_{C_{0}}=\left.T_{C_{0}, C_{F}} \cdot T_{C_{F}, C_{S}} \cdot A\right|_{C_{S}} \cdot
$$

Tracking and calibration software. We rely on two pieces of software for all photogrammetric image processing: Tritop Deformation Software ${ }^{1}$ and i3AxOri, a lab-developed software based on the AxOri photogrammetric bundle adjustment library ${ }^{2}$. Tritop is used to recognize the coded and uncoded points in the images and to compute a first assessment of the position of the cameras. This data is then exported to i3AxOri in which we have more flexibility and control on what we want to compute given our input parameters.

\footnotetext{
${ }^{1}$ Gom, Tritop Deformation Software, http://www.gom.com/3d-software/ tritop-deformation-software.html

${ }^{2}$ Axios 3D, Axori photogrammetric bundle block adjustment, http://www.axios3d.de
} 
Evaluating the registration accuracy. The Gom Atos III acquisition software recognizes any coded or uncoded target present in the scene. In our case the full surface is covered with over 60 uncoded targets of diameter $6 \mathrm{~mm}$. More than 10 targets are visible in each mesh. It is possible to export the list of the targets visible in each mesh. We thus have $\left.A_{i_{j}}\right|_{C_{S}}$ the coordinates of target point $j$ from mesh $i$.

Using equation 1 we calculate $\left.A_{i_{j}}\right|_{C_{0}}$ for all targets of all meshes. For every target $j$ we now calculate

$$
D_{(i, k)_{j}}=\left.A_{i_{j}}\right|_{C_{0}}-\left.A_{k_{j}}\right|_{C_{0}}
$$

for every pair of meshes $(i, k)$ where target $j$ is visible. If the registration were perfect, the result of this subtraction would be a null vector. Since the registration is imperfect, $D_{(i, k)}$, provides us a measure of the accuracy of the registration.

\section{Results and Discussion}

The various acquisitions were performed in the following order:

1. Photogrammetric cameras interior orientation (three days in advance)

2. Tracking frame calibration

3. Acquisition system interior orientation

4. Tracking frame to acquisition system orientation

5. Simultaneous digitization of the car door and photogrammetric tracking of the frame

6. Photogrammetric cameras exterior orientation

We first quickly present the accuracy achieved for the individual calibrations (steps 1 to 4 and 6 ). Then we analyze the accuracy with which we track the target frame (5) and finally we look in to the accuracy of the final registration. All accuracy values (simulations and measurements) are given at $2 \sigma$.

\subsection{Individual calibrations}

The accuracy of the individual calibrations described in section 2.3 is given in table 1. When available, they are compared with the expected accuracy of the simulations. The simulations were run with two levels of noise: low noise, corresponding to a best-case-scenario and higher noise, corresponding to a realistic situation. The accuracy reached is always in between the results for these two configurations.

The tracking cube calibration is performed with much higher accuracy than expected, though this value does not take into account the deformation that the aluminum cube may undergo due to temperature variations for example. 
Table 1: Individual calibrations accuracy compared to expected accuracy from the simulations.

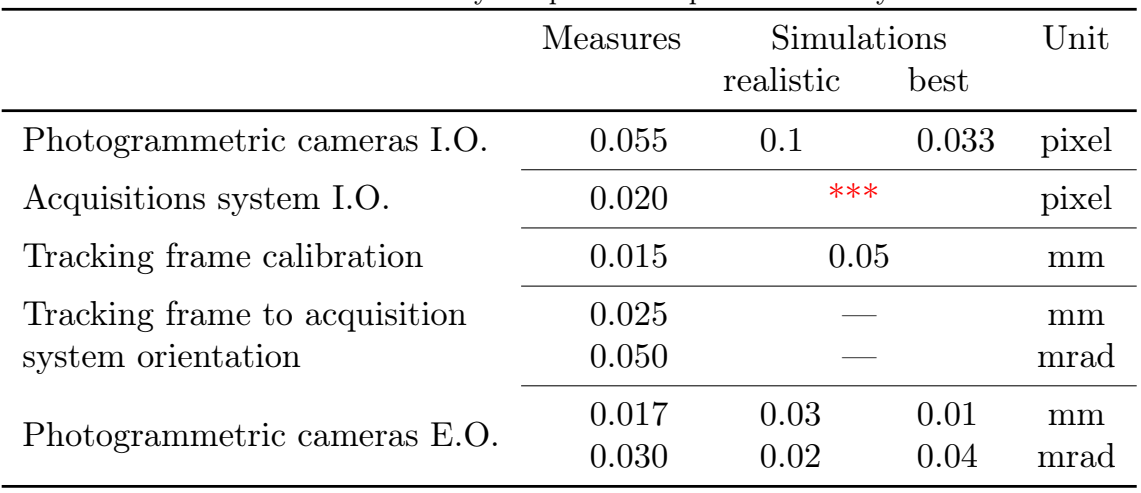

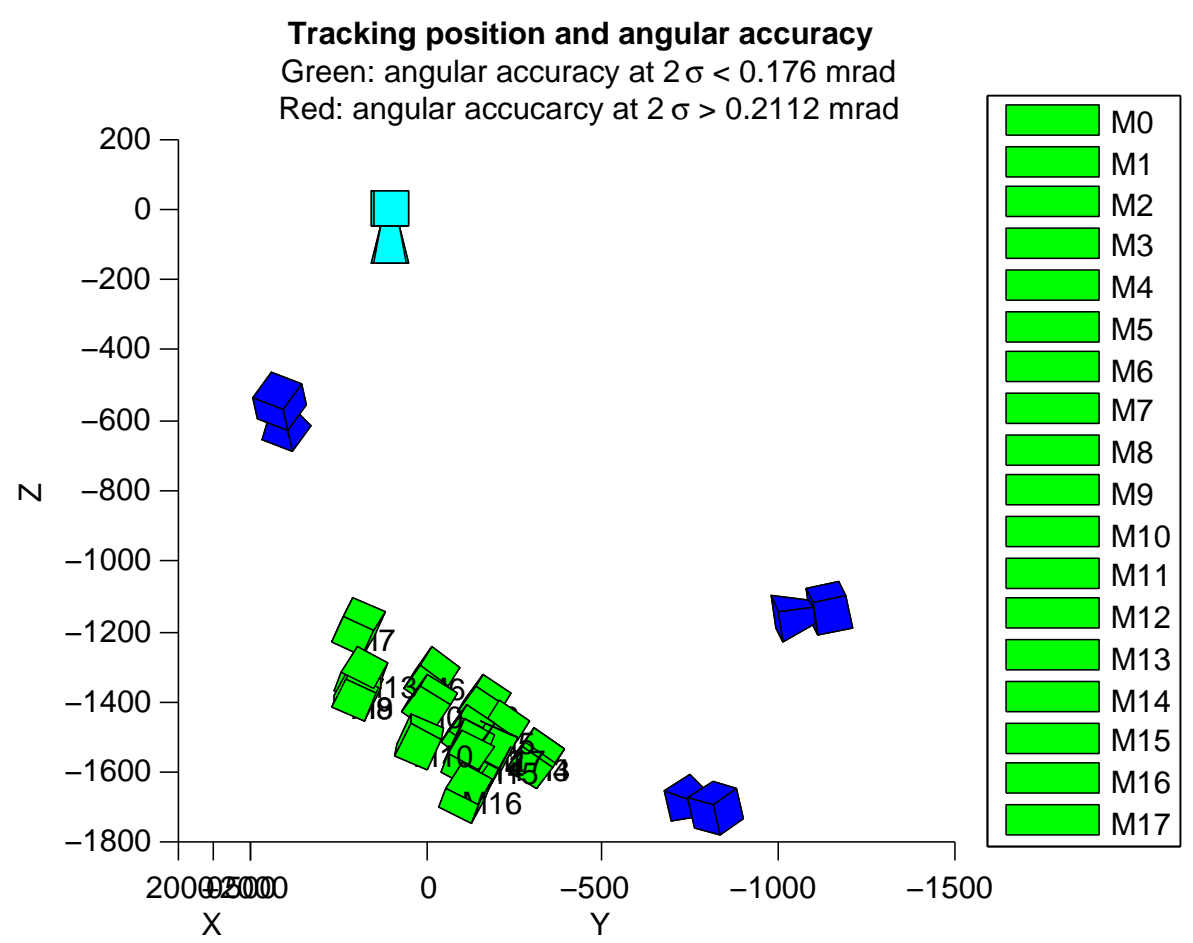

Figure 4: Relative position of the cameras (fixed, blue) and the tracking frame for all acquisitions. *** Redo: colors, orientation, remove text on figure and title 


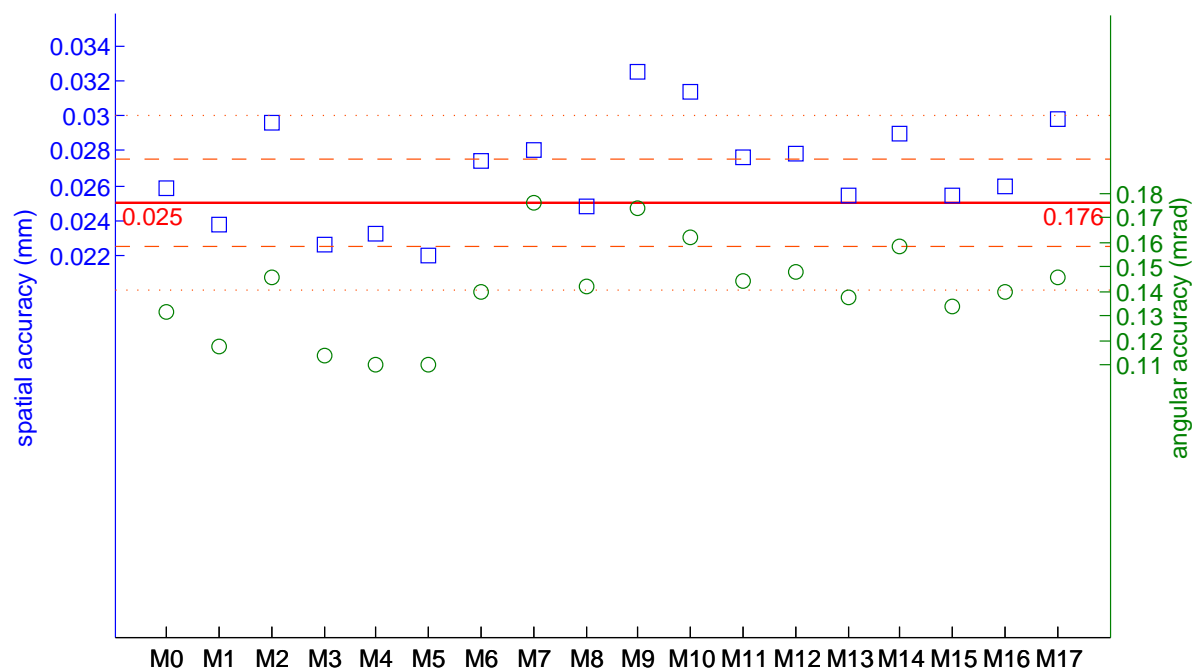

Figure 5: Spatial accuracy (blue squares) and angular accuracy (red circles) of the tracking for all 18 acquisition positions compared to the simulation results of the realistic scenario (red line).

\subsection{Tracking the frame}

Eighteen acquisitions are performed with the 3D digitization system. They are numbered M0 to M17. The relative position and orientation of the four tracking cameras and the tracking cube is illustrated figure 4 .

This subsection examines the accuracy with which we evaluate $T_{C_{0}, C_{F}}$ for each position. The spatial and angular accuracy achieved are compared to the simulation results of the realistic scenario figure 5 . Though the spatial accuracy of the results is not as good as expected, the angular accuracy is always better than the value reached during the simulations.

Compared to the tracking accuracy target fixed by our registration goal $(0.12 \mathrm{~mm}$ spatially and $0.158 \mathrm{mrad})$, the results are quite satisfying: the target spatial accuracy is always reached with a comfortable margin while the angular accuracy is insufficient for only three positions: M7, M9 and M10.

\subsection{Registration}

$* * *$

\section{Conclusion and Perspectives}

- Contactless 3D acquisition and registration

- No need for targets

- Accuracy: *** 
- Independent from sensor

- Different 3D sensors can be used

- Can be extended to 2D projection on 3D model

- Aluminum profiles are fine for rapid prototyping but they are heavy and temperature dependent: a real object should be made of carbon

\section{Acknowledgments}

We would like to thank the Conseil Régional de Bourgogne, France, as well as i3mainz laboratory, Germany, for their funding and support. GOM lent us with a research license for Tritop which was greatly appreciated. Jens Bingenheimer helped perform the measurements. We thank him for his enthusiasm and his meticulosity.

\section{References}

[1] P. J. Besl, N. D. McKay, A Method for Registration of 3-D Shapes, IEEE Transactions on Pattern Analysis and Machine Intelligence 14 (1992) 239256.

[2] S. Rusinkiewicz, M. Levoy, Efficient variants of the ICP algorithm, in: Proceedings of the Third International Conference on 3-D Digital Imaging and Modeling, Quebec City, Quebec, Canada, pp. 145-152.

[3] J. Hefele, Real-time photogrammetric algorithms for robot calibration, International Archives of Photogrammetry and Remote Sensing XXXIV (2002) $33-38$.

[4] T. Clarke, X. Wang, The control of a robot end-effector using photogrammetry, International Archives of Photogrammetry and Remote Sensing XXXIII (2000) 137-142.

[5] R. Schütze, C. Raab, F. Boochs, H. Wirth, J. Meier, Optopose - a multicamera system for fast and precise determination of position and orientation for moving effector, in: 9th Conference on Optical 3D Measurement Techniques, Vienna, Austria.

[6] H.-G. Maas, Dynamic photogrammetric calibration of industrial robots, in: Proceedings of SPIE Vol 3174 Videometrics V, SPIE, San Diego, CA, USA, 1997, pp. 106-112.

[7] C. Simon, R. Schütze, F. Boochs, F. S. Marzani, Asserting the Precise Position of 3D and Multispectral Acquisition Systems for Multisensor Registration Applied to Cultural Heritage Analysis, in: K. Schoeffmann, B. Merialdo, A. G. Hauptmann, C.-W. Ngo, Y. Andreopoulos, C. Breiteneder (Eds.), MMM, volume LNCS 7131, Springer-Verlag Berlin Heidelberg, Klagenfurt (Austria), 2012, pp. 597-608. 